\title{
COVID-19 Health Equity Dashboard - Addressing Vulnerable Populations
}

\author{
Star Liu Emily Wall Shivani A. Patel* Yubin Park ${ }^{\dagger}$
}

\begin{abstract}
We present a case study of the COVID-19 Health Equity Dashboard, an open-source ${ }^{1}$ web-based interactive data visualization, that provides timely, localized, and actionable data of the ongoing COVID-19 pandemic ${ }^{2}$. The dashboard features interactive maps and charts alongside population vulnerability characteristics, allowing for benchmarking county-level outcomes and disparities against the state and nation. While the dashboard faces several public health communication challenges, we continue to investigate and support data dissemination for public health officials' decision making.
\end{abstract}

\section{BACKGROUND}

COVID-19 first emerged in the United States in late January 2020 and soon became a national and global pandemic. By March and April, more data has become available daily, including hospitalization rates and patient characteristics. In an early April issue of the Morbidity and Mortality Weekly Report, findings suggested that black populations could be impacted disproportionately by COVID19 [1]. Hence, we were motivated to investigate how underlying social characteristics could act as precursors to population-level vulnerability and how such vulnerabilities may inform targeted response to differential health impacts. By late April, we published the dashboard, utilizing data aggregated by the New York Times, the US Centers for Disease Control and Prevention, and other groups.

Health Equity. Health equity refers to the principle that "everyone has a fair and just opportunity to be as healthy as possible"3. Promoting health equity requires enacting policies that cater to the needs of vulnerable populations [2]. As COVID-19 impacts each community differently and decisions are implemented locally, it becomes imperative to address differential risk across communities and benchmark outcomes.

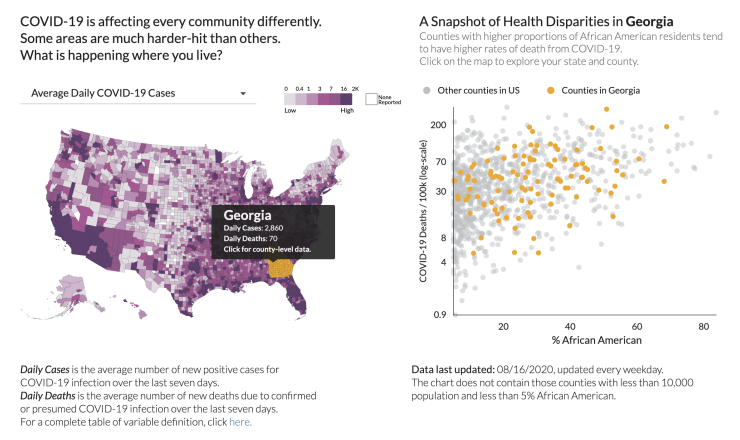

Figure 1: The COVID-19 Health Equity Dashboard.

*e-mail: \{star.liu, emily.wall, s.a.patel\}@emory.edu

†e-mail: yubin@bonsairesearch.com

\footnotetext{
${ }^{1}$ https://github.com/yubin-park/emory-covid19

${ }^{2}$ https://covid19. emory.edu

${ }^{3}$ https://www.rwjf.org/en/library/research/2017/05/ what-is-health-equity-.html
}

\section{DEsign Considerations}

Interface Scheme. The dashboard includes a variety of data comparing health equity in the COVID-19 pandemic; hence, to provide a simple summary and easy navigation of the dashboard, our design follows the Information-Seeking Mantra: "overview first, zoom and filter, then details-on-demand" [3]. The dashboard begins at the national level for contextual overview. With one click, the user is taken to the state level data. Here, the progression in the state is shown over time with respect to daily cases, percentage of positive cases, and percentage of occupied beds, as well as cases compared against the state's race distribution. A second click navigates to the county page, where users are pointed to metrics, such as the total, average, and per capita cases and deaths, shown in tables and trendlines; and the county's impact on case rates by factors like race and income are compared against other counties in scatterplots.

Actionable Data. Given the differential impact of COVID-19 across counties and the role of county health officials in epidemic response, we chose to position maps and charts next to population characteristics for quick comparisons and data contextualization. On closer inspection, one design consideration distinct from other dashboards is benchmarking local outcomes and population characteristics such as race and comorbidities, realized in line and bar graphs. Based on feedback from epidemiologists and clinicians, design iterations of the dashboard added new metrics of COVID-19 impacts such as the percentage of positive cases and the percentage of beds occupied by COVID-19 patients to states' summary statistics. By meeting field requests, we make relevant data easily accessible for decision makers to target actions towards vulnerable communities.

\section{ONGOING AND FUTURE WORK}

With continued feedback from researchers, epidemiologists, clinicians, and laypersons, we actively enhance the dashboard functionality and reporting of metrics in order to effectively communicate key statistics. Focusing on measures of vulnerability may mobilize resources and attention for change. In fact, we are building a standalone dashboard for the Georgia Department of Public Health to disseminate detailed impacts of COVID-19 by demographic subgroup at sub-county levels. Using Natural Language Processing, we plan to develop narratives alongside the data visualizations to provide users with interpretable and actionable synthesis of statistical data. While challenges remain, we argue that an equity-focused dashboard can provide invaluable data-driven support in this fight.

\section{ACKNOWLEDGMENTS}

This work was supported by the Woodruff Health Sciences Center and the Robert Wood Johnson Foundation.

\section{REFERENCES}

[1] S. Garg et al. Hospitalization rates and characteristics of patients hospitalized with laboratory-confirmed coronavirus disease 2019 - covid-net, 14 states, march 1-30, 2020. April 2020. https://www.cdc.gov/ mmwr/volumes/69/wr/mm6915e3.htm.

[2] T. S. Purnell et al. Achieving health equity: Closing the gaps in health care disparities, interventions, and research. DISPARITIES, HOSPITAL FINANCING \& MORE, 35(8), August 2016. doi: 10.1377/hlthaff.2016. 0158

[3] B. Shneiderman. The eyes have it: A task by data type taxonomy for information visualizations. In Proceedings 1996 IEEE symposium on visual languages, pp. 336-343. IEEE, 1996. 